\title{
CUBIC INTERPOLATION BASED CHANNEL ESTIMATOR FOR RECTANGULAR $M$-QAM
}

\author{
0. O. Ogundile ${ }^{1, *}$, O. A. Osanaiye ${ }^{2}$ and E. O. Falayi ${ }^{3}$

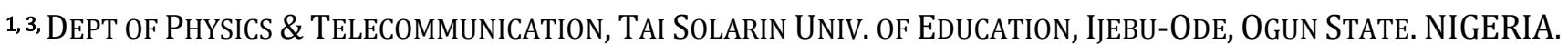 \\ 2 Department of Telecommunication, Federal University of TeChnology Minna, Niger State, NigERIA. \\ E-mail addresses:1 ogundileoo@gmail.com, 2 opeyemi.osanaiye@alumni.uct.ac.zn, \\ 3 olukayodefalayi@yellow.com
}

\begin{abstract}
This paper propose a Pilot Symbol Assisted Modulation (PSAM) channel estimation and compensation technique for rectangular M-QAM based on Cubic interpolation in order to determine the channel state information (CSI) over flat Rayleigh fading channels. The proposed Cubic channel estimator technique is based on sliding window approach and pilot symbol estimates adjustment in order to reduce its computational time complexity. The Cubic estimator is combined with the Koetter and Vardy (KV) Reed-Solomon (RS) decoder to test its performance. The simulation results show that the Cubic interpolation gives the same performance as the Linear, and Sinc interpolators over slow flat Rayleigh fading channel; however, it achieves significant performance improvement of $+1.0 d B$ in symbol error rate (SER) over fast flat Rayleigh fading channel.
\end{abstract}

Keywords: CSI, cubic estimator, fading channel, M-QAM, PSAM

\section{INTRODUCTION}

Pilot Symbol Assisted Modulation (PSAM) channel estimation techniques have been used extensively and effectively in practical estimation of the channel state information (CSI) for $M$-ary quadrature amplitude modulation ( $M$-QAM) systems over Rayleigh fading channels with Additive White Gaussian Noise (AWGN). In practice, the CSI is not available to the demodulator; therefore, different channel estimation techniques have been proposed in literature to analyse the effect of channel errors and improve the performance of the demodulator [1-7]. Emphasis has been on the trade-off between the complexity and performance of these channel estimation techniques over flat Rayleigh fading channels.

With high data transmission rate demand and reduced bandwidth consumption in wireless technology, the use of spectral efficient and high data rate modulation schemes is of practical importance. For high $M, M$-QAM systems achieve high throughput without additional bandwidth expansion. However, the performance of $M$ QAM over Rayleigh fading channels is dependent on pinpoint accurate estimations of the CSI. In [2], a PSAM channel estimation technique using Gaussian interpolation to estimate the CSI was proposed. Although, the Gaussian interpolation method is simple with low complexity, the performance of the estimator rapidly degrades with increase in the frame length size $L$. The author in [1] and [3] employed Wiener filtering interpolation and Sinc interpolator respectively to estimate the CSI. Both methods give comparable symbol error rate (SER) performance at small Doppler frequencies but the Wiener interpolation comes with increased complexity as it requires priori information of the channel in order to estimate the filter coefficients [3]. The Sinc interpolator method yields better SER performance over flat Rayleigh fading channel as compared to Gaussian interpolation; however, the performance of these estimation techniques degrade rapidly as the Doppler frequency increases.

CSI estimates for $M$-QAM over flat Rayleigh fading channels using Linear interpolation were analysed in [6] and [8]. The Linear interpolation PSAM channel estimation technique achieves considerable good SER performance over fast flat Rayleigh fading channel. This interpolation technique offers low computational time complexity compared to most interpolation techniques found in literature. The performance of this interpolation technique degrades with increase in $L$ but the technique is very useful when low implementation complexity is key to the system design. Since there should be a balance between the performance of the 
channel estimation technique and its implementation complexity, this work proposes an estimation technique for rectangular $M$-QAM based on Cubic interpolation. The proposed Cubic channel estimator is based on a sliding window approach and pilot symbol estimates adjustment in order to reduce the computational time complexity of the estimation technique.

The rest of the paper is structured as follows. In Section 2.0, the general system model for the channel estimators and notations are explained. A brief overview at the existing PSAM channel estimation techniques is carried out in Section 3.0. Section 4.0 explains the proposed Cubic channel estimator for rectangular $M$-QAM in detail. Comparison and discussion of results are presented in Section 5.0. Section 6. 0 summarises and conclude the paper.

\section{SYSTEM MODEL}

Consider the PSAM system model shown in Figure 1. The input data is encoded using an $R S(15,7)$ low rate code. The encoded data is mapped to rectangular 16QAM complex data symbols in which both the real and imaginary parts take values from a set $( \pm 1 c, \pm 3 c, \ldots, \pm c(\Gamma-1))$, where $\Gamma$ is of the form $M=$ $\Gamma^{2}$ [9]. Known pilot symbols $\left(d_{1}, d_{2}, \ldots d_{\hat{p}}\right)$ are periodically inserted to the transmitted QAM symbols as shown in Figure 2. The total number of pilot symbols inserted in each transmitted codeword $n$ is defined by $\hat{p}$. The frame number, and total number of frames in each transmitted codeword $n$ are denoted as $f$, and $f+i$ respectively (note, that $i$ is the total number of frames). Each frame starts with a pilot symbol and subsequently $L-1$ data symbols. The data and pilot symbols are transmitted over a flat Rayleigh fading channel with a Doppler frequency $f_{D}$.

The faded received signal corrupted with AWGN in the current $\bar{m} t h$ frame after down-converting and matched filtering is given as:

$$
\bar{r}_{\bar{m}}^{\bar{s}}=\bar{h}_{\bar{m}}^{\bar{s}} \bar{t}_{\bar{m}}^{\bar{s}}+z_{\bar{m}}, \quad \bar{s}=1, \cdots, L-1,
$$

where $\bar{h}_{\bar{m}}^{\bar{s}}$ is the complex zero mean Gaussian variables denoting the fading distortion at the data symbol positions, $z_{\bar{m}}$ is the complex AWGN with variance $N_{o} / 2$, and $\bar{t}_{\bar{m}}^{\bar{s}}$ is the transmitted QAM symbols.

The noisy fading estimates at the $\bar{m} t h$ pilot symbol positions is obtained by dividing the received pilot symbols by the known transmitted pilots as:

$$
h_{\bar{m}}=\frac{\hat{r}_{\bar{m}}}{\hat{t}_{\bar{m}}}=\hat{h}_{\bar{m}}+\frac{z_{\bar{m}}}{\hat{t}_{\bar{m}}}
$$

where $\hat{t}_{\bar{m}}$ and $\hat{r}_{\bar{m}}$ are the transmitted and received pilot symbols respectively, $\hat{h}_{\bar{m}}$ is the complex zero mean Gaussian variables representing the fading distortion at the pilot symbol positions. The fading estimates $h_{\bar{m}}^{\bar{s}}$ in the current $\bar{m}$ th frame at the data symbol position is computed by interpolating the known pilot symbol fading estimates $h_{\bar{m}}$.

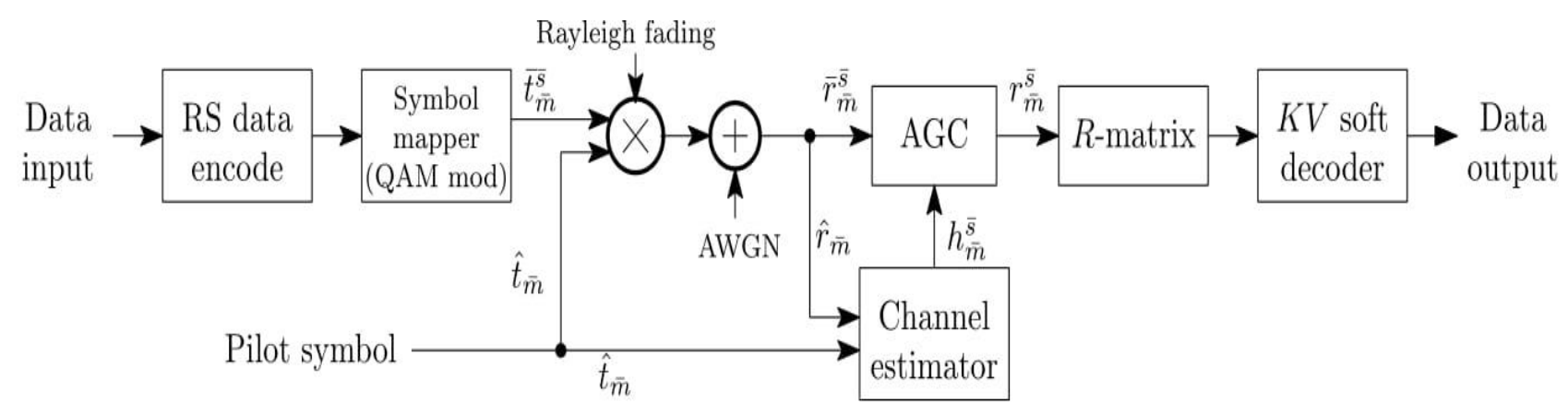

Figure 1: PSAM channel estimator system model.

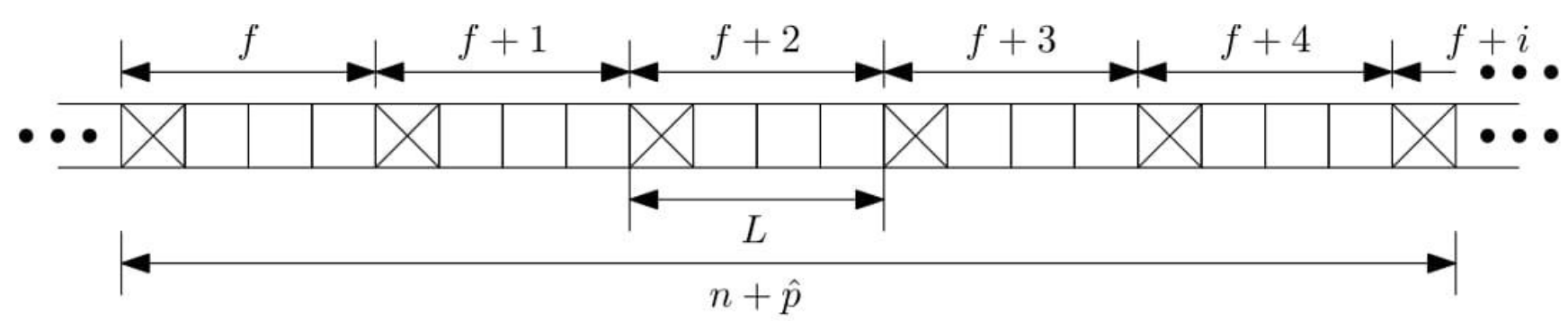

Figure 2: Transmitted $n+\hat{p}$ frame structure. 
The received data symbol $\bar{r}_{\bar{m}}^{\bar{s}}$ is scaled to compensate for the channel error in a process called Automatic Gain Control (AGC). This is achieved by dividing the received data symbol $\bar{r}_{\bar{m}}^{\bar{s}}$ by the fading channel estimates $h_{\bar{m}}^{\bar{s}}$ as:

$$
r_{\bar{m}}^{\bar{s}}=\frac{\bar{r}_{\bar{m}}^{\bar{s}}}{h_{\bar{m}}^{\bar{s}}}
$$

where $r_{\bar{m}}^{\bar{s}}$ is the scaled received data symbol. The scaled received data symbols $r_{\bar{m}}^{\bar{s}}$ are used in computing the $R$ matrix which is fed into the KV soft decoder [10]. The $\mathrm{KV}$ algorithm converts the received soft probabilistic reliability information from the channel into a choice set of interpolation points along with their multiplicities in the Guruswami and Sudan algorithm [10] [11] [12]. A list size $\bar{L}=4$ is assumed for the KV soft decoder [10] in the system model of Figure 1 for reduced simulation complexity. The list size $\bar{L}(\bar{L} \geq 1)$ determines the decoding performance of the KV softdecision decoder. The higher the value of the list size the better the decoding performance of the $\mathrm{KV}$ algorithm but at the price of increased decoding complexity.

\section{PSAM INTERPOLATION TECHNIQUES}

\subsection{Linear Interpolation}

The Linear interpolation based channel estimation for $M$-QAM offers very low computational time complexity $(O(n))$ to the overall system design. Basically, the interpolation process to estimate the CSI at the data symbol positions is achieved by using the fading estimates $h_{\bar{m}}$ of two nearest pilot symbols. Considering the transmitted frame structure $(L=4)$ of Figure 2 , the CSI at the $\bar{s} t h$ data symbol positions in the current $\bar{m} t h$ frame is given as:

$$
h_{\bar{m}}^{\bar{s}}=\sum_{\bar{s}=1}^{L-1} h_{\bar{m}}+\gamma_{l}\left(h_{\bar{m}+1}-h_{\bar{m}}\right)
$$

where $\gamma_{l}$ is the Linear interpolation coefficient defined as $\bar{s} / L$.

\subsection{Sinc Interpolation}

Sinc interpolation circumvents the computational load inherent in the Wiener filtering interpolation. This technique do not require priori information of the channel to estimate the $\operatorname{CSI} h_{\bar{m}}^{\bar{s}}$ at the data symbol positions. The CSI $h_{\bar{m}}^{\bar{s}}$ at the data symbol positions is determined using $I_{o}$ nearest pilot symbol fading estimates $h_{\bar{m}}$, where $I_{o}$ is the interpolation order. Depending on the interpolation order, the Sinc interpolation technique uses $\left\lfloor\left(I_{o}-1\right) / 2\right\rfloor$ previous pilot fading estimates $h_{\bar{m}-\left\lfloor\left(I_{O}-1\right) / 2\right\rfloor}$ the current pilot fading estimate $\left(h_{\bar{m}}\right)$ and $\left\lfloor I_{o} / 2\right\rfloor$ next pilot fading estimates $h_{\bar{m}-\left\lfloor I_{o} / 2\right\rfloor}$ to compute the $\bar{s} t h$ data symbol in the $\bar{m} t h$ frame [4]. Thus, the estimated fading distortion in the $\bar{m} t h$ frame is given as:

$$
h_{\bar{m}}^{\bar{s}}=\sum_{\bar{s}=\left\lfloor\left(I_{o}-1\right) / 2\right\rfloor}^{\left\lfloor I_{o} / 2\right\rfloor} h_{\bar{m}} \gamma_{s}
$$

where $\gamma_{S}$ is the Sinc interpolator coefficient defined as Sinc $\left(\bar{s} / L-I_{o}\right)[3]$, [4]. The interpolation coefficient is weighted with a window function. An interpolation order $I_{o}=4$ is assumed in this paper, and a Hamming window is applied to the Sinc interpolation coefficient [7]. Note, as mentioned in [7], the computational time complexity of the Sinc interpolator increases with increase in the interpolation order $I_{o}$, which subsequently delays the interpolation process.

\section{PROPOSED CUBIC INTERPOLATION}

It is natural to maintain a balance between the performance of the channel estimation technique and its implementation complexity. This work propose a Cubic polynomial channel estimation technique based on Least Square (LS) approximation. The $\operatorname{CSI} h_{\bar{m}}^{\bar{s}}$ at $\bar{m} t h$ data symbol position using this proposed interpolation technique is computed by interpolating the four nearest pilot symbol fading estimates $h_{\bar{m}}$.

Given that $\left(h_{\bar{m}}, h_{\bar{m}+3}\right)$ are the four nearest pilot symbol fading estimates to the $\bar{s}$ th data symbol and $\left(P_{\bar{m}}, \ldots, P_{\bar{m}+3}\right)$ are the known positions of the pilot symbols in the transmitted frame structure as shown in Figure 2. Thus, we compute the fading estimates in the current $\bar{m} t h$ frame as:

$$
h_{\bar{m}}^{\bar{s}}=\sum_{\bar{s}=1}^{L-1} \gamma_{1}+\gamma_{2}(\bar{s})+\gamma_{3}(\bar{s})^{2}+\gamma_{4}(\bar{s})^{3},
$$

where $\left(\gamma_{1}, \ldots, \gamma_{4}\right)$ are the Cubic interpolation coefficients which we define as:

$$
\left[\begin{array}{c}
h_{\bar{m}} \\
h_{\bar{m}+1} \\
h_{\bar{m}+2} \\
h_{\bar{m}+3}
\end{array}\right]=\left[\begin{array}{lllr}
1 & P_{\bar{m}} & P_{\bar{m}}^{2} & P_{\bar{m}}^{3} \\
1 & P_{\bar{m}+1} & P_{\bar{m}+1}^{2} & P_{\bar{m}+1}^{3} \\
1 & P_{\bar{m}+2} & P_{\bar{m}+2}^{2} & P_{\bar{m}+2}^{3} \\
1 & P_{\bar{m}+3} & P_{\bar{m}+3}^{2} & P_{\bar{m}+3}^{2}
\end{array}\right]\left[\begin{array}{l}
\gamma_{1} \\
\gamma_{2} \\
\gamma_{3} \\
\gamma_{4}
\end{array}\right]
$$

This proposed technique experiences little delay with increase in $L$ but the computational time complexity remains the same for change in the frame length size $L$, and all defined parameters of the form of Eqs. (6) and (7). Thus, the computational time complexity of this Cubic interpolation technique is independent of $L$ nor any input parameter of the form of Eqs. (6) and (7).

However, executing Eq. (7) to compute the cubic interpolation coefficients $\left(\gamma_{1}, \ldots, \gamma_{4}\right)$ add a computational time complexity of $\left(O\left(n^{3}\right)\right)$ to proposed cubic channel 
estimator for large input data. Therefore, a sliding window approach is assumed in computing the cubic interpolation coefficients in order to reduce the computational time complexity from $\left(O\left(n^{3}\right)\right) \rightarrow$ $\left(O\left(n^{2}\right)\right)$.

The four nearest pilot symbols positions are fixed as $\left(P_{1}, P_{1+L}, P_{1+2 L}\right.$, and $\left.P_{1+3 L}\right)$, and we shift (adjust) the fading estimates at the pilot symbol positions from $h_{\bar{m}}$ to $h_{\bar{m}+1}$ while computing the interpolation coefficients from frame $f$ to $f+i$. Thus, Eq. (7) is modified as:

$$
\left[\begin{array}{c}
h_{\bar{m}} \\
h_{\bar{m}+1} \\
h_{\bar{m}+2} \\
h_{\bar{m}+3}
\end{array}\right]=\left[\begin{array}{cccc}
1 & 1 & 1 & 1 \\
1 & P_{1+L} & P_{1+L}^{2} & P_{1+L}^{3} \\
1 & P_{1+2 L} & P_{1+2 L}^{2} & P_{1+2 L}^{3} \\
1 & P_{1+3 L} & P_{1+3 L}^{2} & P_{1+3 L}^{2}
\end{array}\right]\left[\begin{array}{c}
\gamma_{1} \\
\gamma_{2} \\
\gamma_{3} \\
\gamma_{4}
\end{array}\right]
$$

In order to reduce the computational complexity in computing the interpolation coefficients. As shown in Figure 2, each frame starts with a pilot symbol, so $P_{1}$ is always equal to one $\left(P_{1}=1\right)$ for any transmitted structure frame length size $L$. Using Eqs. (8) and (6), we can estimate the fading distortion at the data symbol positions in the current $\bar{m} t h$ frame.

\section{SIMULATION RESULTS}

To verify the performance of the proposed Cubic estimator technique, the estimator is tested with rectangular 16-QAM combined with the KV soft decoder [10]. The system is simulated using MATLAB software. The estimator is applied assuming a slowvarying flat Rayleigh fading channel (Doppler frequency $f_{D}=50 \mathrm{~Hz}$ ), and a fairly fast flat Rayleigh fading channel (Doppler frequency $f_{D}=100 \mathrm{~Hz}$ ) as shown in Figures 3 and 4, respectively. Frame length sizes $L=4$ and $\mathrm{L}=6$ are used in order to test the performance of the cubic estimator for increase in $L$. All other parameters for each channel estimation technique are defined as above.

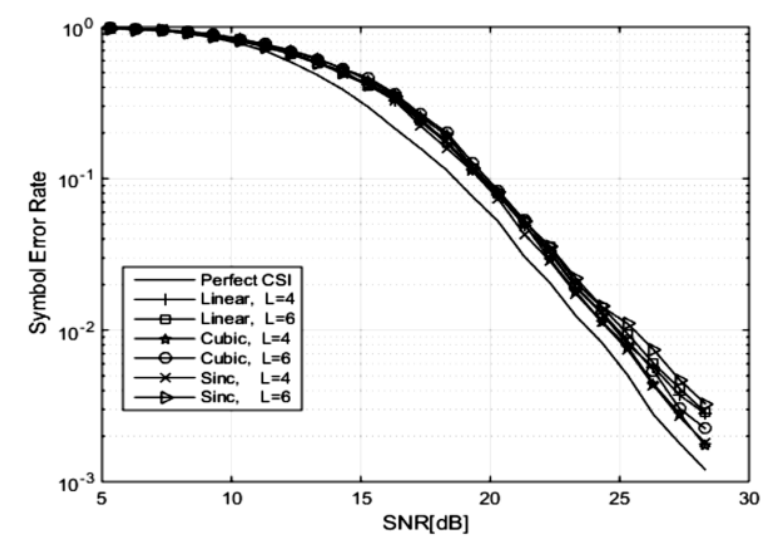

Figure 3: SER performance comparison for different PSAM channel interpolators and frame length sizes $L$ for rectangular 16-QAM combined with KV soft decoder over slow flat Rayleigh fading channel. Doppler frequency, $f_{D}=50 \mathrm{~Hz}$, and $\bar{L}=4$.
As shown in Figure 3, the Cubic channel estimator offers comparable SER performance with the Linear, and Sinc channel estimators, even with increase in $L$. However, the performance of the Linear, and Sinc interpolators degrade with increase in the Doppler frequency $f_{D}$ (from $f_{D}=50 \mathrm{~Hz}$ to $f_{D}=100 \mathrm{~Hz}$ ), more significantly when $L$ increases (from $L=4$ to $L=6$ ) as depicted in Figure 4. The proposed Cubic estimator offers significant improvement in SER performance $(+1.0 d B)$ as compared to the Linear, and Sinc interpolators over fast-varying flat Rayleigh fading channel. Also as shown in Figure 4, at SNR $>15 d B$, it is observed that the performance of the Cubic interpolator at $L=6$, surpasses the Linear interpolator performance by $+1.0 \mathrm{~dB}$, and the Sinc interpolator performance by $+0.3 d B$ at frame length size $L=4$. The cubic interpolator adds delay to the interpolation complexity time as it requires four nearest pilot fading estimates $\left(h_{\bar{m}}, \ldots, h_{\bar{m}+3}\right)$ to calculate the CSI. The proposed Cubic estimator provides a practical computational time complexity $\left(O\left(n^{2}\right)\right)$ to the overall system model for large input data size compared to the time complexity $(O(n))$ provided by the Linear, and Sinc interpolation (complexity of the Sinc interpolator increases with increase in $I_{o}$, which may become more complex than the Cubic interpolation technique for high $I_{o}$ ). The Cubic interpolator offers a SER gain of $+1.0 d B$ over the Linear, and Sinc channel estimators at $S N R>15 d B$ assuming a fast flat Rayleigh fading channel. Thus, the proposed estimation technique for rectangular $M$-QAM offers a reasonable balance in the channel estimator performance and complexity, and it can be implemented for real time systems.

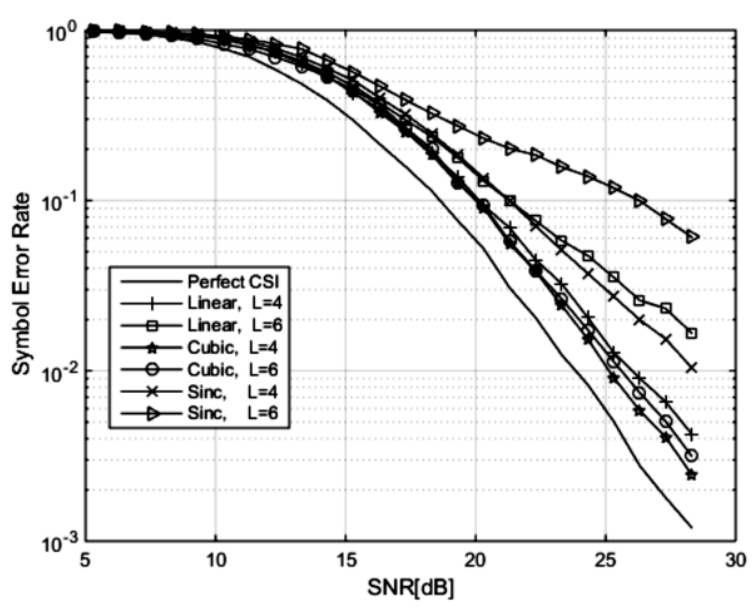

Figure 4: SER performance comparison for different PSAM channel interpolators and frame length sizes $L$ for rectangular 16-QAM combined with KV soft decoder over fast flat Rayleigh fading channel. Doppler frequency, $f_{D}=100 \mathrm{~Hz}$, and $\bar{L}=4$. 


\section{CONCLUSION}

The PSAM channel estimation for rectangular $M$-QAM using Linear, Sinc, and Cubic interpolation techniques combined with KV soft decoder has been analysed for different frame length sizes $L$. The proposed Cubic channel estimator exhibits considerably higher complexity than the Linear, and Sinc estimators, but it offers superior SER performance to the Linear, and Sinc estimators over fast flat fading channel. Furthermore, at high signal to noise ratio (SNR $>25 d B$ ), it can be observed from the simulation results that the Cubic estimator gives significant SER gain performance $(+1.5 \mathrm{~dB})$ as compared to the other channel estimators analysed in the paper over fast flat Rayleigh fading channel. Simulation results of the SER performance for the different channel estimators confirm our analysis.

\section{REFERENCES}

[1] J. Cavers, "An Analysis of Pilot Symbol Assisted Modulation for Rayleigh Fading Channels," IEEE, Transaction on Vehicular Technology, vol. 40, no. 4, pp. 686-693, November 1991.

[2] S. Sampei and T. Sunaga, "Rayleigh Fading Compensation for QAM in Land Mobile Radio Communications," IEEE Transaction on Vehicular Technology, vol. 42, pp. 137-146, May 1993.

[3] Y. Kim, C. Kim, G. Jeong, Y. Bang, H. Park and S. Choi, "New Rayleigh fading channel estimator based on PSAM channel sounding technique," Communications, 1997. ICC '97 Montreal, Towards the Knowledge Millennium. 1997 IEEE International Conference on, Montreal, Que., pp. 1518-1520 vol.3, 1997.

[4] X. Tang, M. S. Alouini and A. J. Goldsmith, "Effect of channel estimation error on M-QAM BER performance in Rayleigh fading," in IEEE
Transactions on Communications, vol. 47, no. 12, pp. 1856-1864, Dec 1999.

[5] O. Oyerinde and S. Mneney, "Iterative receiver with soft-input-based-channel estimation for orthogonal frequency division multiplexinginterleave division multiple access systems," Communications, IET, vol. 8, no. 14, pp. 24452457, Sept 2014.

[6] M. Benjillahi and L. Szczeciński, "Low Complexity Channel Estimation with Pilot Symbol Assisted Modulation," IEEE Signal Processing and Its Applications, vol. 2, pp. 471-474, August 2005.

[7] L. Xiao, X. Dong, and A. C. K. Soong, "On the Design of Sinc Interpolator for Pilot Symbol Assisted Modulation Systems," IEEE Transaction on Wireless Communication, vol. 5, no. 9, September 2006.

[8] A. Mämmelä and V. Kaasila, "Smoothing and Interpolation in a Pilot Symbol Assisted Diversity System," International Journal of Wireless Information Networks, vol. 4, no. 3, 1997.

[9] O. Ogundile and D. Versfeld, "Performance evaluation of polynomial based channel estimation for OFDM systems on time-varying frequency-selective fading channels," in AFRICON, Sept 2015, pp. 1-6. 2015,

[10] R. Koetter and A. Vardy, "Algebraic soft-decision decoding of Reed-Solomon codes," IEEE Transactions on Information Theory, vol. 49, no. 11, pp. 2809-2825, Nov 2003.

[11] V. Guruswami and M. Sudan, "Improved decoding of reed-Solomon and algebraic-geometry codes," IEEE Transactions on Information Theory, vol. 45, no. 6, pp. 1757-1767, Sep 1999.

[12] M. Sudan, "Decoding of Reed-Solomon Codes Beyond the Error Correction Bound." J. Complexity, vol. 12, pp. 180-193, 1997. 